\title{
Mathematical model for the ubiquitin activating enzyme E1
}

\author{
Francisco Javier López-Cánovas ${ }^{1}$, Francisca Cánovas ${ }^{2}$, María Antonia Günther Sillero ${ }^{1}$, Antonio Sillero ${ }^{1}$
}

${ }^{1}$ Departamento de Bioquímica, Instituto de Investigaciones Biomédicas Alberto Sols UAM/CSIC, Facultad de Medicina, Madrid, Spain;

${ }^{2}$ Departamento de Matemáticas e Informática, Escuela Técnica Superior de Ingenieros de Caminos, Canales y Puertos, Universidad Politécnica de Madrid, Madrid, Spain.

Email: antonio.sillero@uam.es

Received 21 December 2009; revised 4 January 2010; accepted 8 January 2010.

\begin{abstract}
The ubiquitin-activating enzyme E1 (EC 6.3.2.19) represents the first step in the degradation of proteins by the ubiquitin proteasome pathway. E1 transfers ubiquitin from the ubiquitinated E1 to the ubiquitin carrier proteins (E2), ubiquitin-protein ligases (E3) and proteins. This process is rather complex, and known from the work of Haas, Ciechanover, Hershko, Rose and others. The occurrence of 19 hypothetical intermediate enzyme forms (EFs) and 22 different reactions were considered in the presence of ubiquitin (Ub), ATP, adenosine 5'-tetraphosphate $\left(\mathrm{p}_{4} \mathrm{~A}\right)$, pyrophosphate $\left(P_{2}\right)$, and tripolyphosphate $\left(P_{3}\right)$ as substrates, and iodoacetamide (IAA) and dithiothreitol (DTT) as inhibitors. Inspired by the work of Cha (Cha (1968) J. Biol. Chem., 243, 820-825) we have treated these reactions in two complementary ways: in rapid equilibrium and in steady state. The kinetics of both types of reactions were simulated and solved with a system of ordinary differential equations using the Mathematica Program. The ubiquitination of $\mathrm{E} 1$ has been also theoretically coupled to the ubiquitination of E2, E3 and proteins. This makes the model useful to predict the theoretical influence of inhibitors (or of changes in some parameters of the reaction) on the ubiquitination of proteins. The Program responds to changes in the concentration of ATP or ubiquitin and has predictive properties as shown by the influence of AMP on the synthesis of $p_{4} A$, calculated theoretically and confirmed experimentally.
\end{abstract}

Keywords: Dinucleoside Polyphosphates; Adenosine Tetraphosphate; Tripolyphosphate; Proteasome; Mathematical Model

\section{INTRODUCTION}

The work here presented deals with the development of a mathematical model for the ubiquitin activating en- zyme E1 (EC 6.3.2.19) and is based on the following grounds, A, B, C, D:

1) Ligases catalyze the synthesis of diadenosine tetraphosphate $\left(\mathrm{Ap}_{4} \mathrm{~A}\right)$ and other (di)nucleoside polyphosphates through the general reactions a) and b), where $\mathrm{X}$ is a co-substrate of a ligase [1]

a) $\mathrm{E}+\mathrm{X}+\mathrm{ATP}<----->\mathrm{E}-\mathrm{X}-\mathrm{AMP}+\mathrm{P}_{2}$

b) $\mathrm{E}-\mathrm{X}-\mathrm{AMP}+\mathrm{ATP}---->\mathrm{Ap}_{4} \mathrm{~A}+\mathrm{X}+\mathrm{E}$

As reaction a) is reversible, the synthesis of $A_{4} A$ is greatly favored in the presence of pyrophosphatase (PPase). In our experience, reaction b) is rather unspecific and the AMP residue of the E-X-AMP complex may react with the terminal phosphate of almost any molecule containing an intact terminal pyrophosphate, such as ATP, GTP, adenosine tetra phosphate $\left(\mathrm{p}_{4} \mathrm{~A}\right)$, tri, tetra, penta phosphate $\left(\mathrm{P}_{3}, \mathrm{P}_{4}, \mathrm{P}_{5}\right)[2]$.

2) The ubiquitin-activating enzyme $\mathrm{E} 1$ is a particular ligase using ubiquitin (Ub) and ATP (reaction c), or $\mathrm{p}_{4} \mathrm{~A}$ (reaction d) as substrates for the synthesis of the AMP derivative of the enzyme and, by the same token, uses $\mathrm{P}_{2}$ or $\mathrm{P}_{3}$ in the reverse reactions; $\mathrm{p}_{4} \mathrm{~A}$ and $\mathrm{P}_{3}$ act as analogs of ATP and $\mathrm{P}_{2}$, respectively [3].

c) $\mathrm{E}+\mathrm{Ub}+\mathrm{ATP}<----->$ E-Ub-AMP $+\mathrm{P}_{2}$

d) $\mathrm{E}+\mathrm{Ub}+\mathrm{p}_{4} \mathrm{~A}<----->\mathrm{E}-\mathrm{Ub}-\mathrm{AMP}+\mathrm{P}_{3}$

The reactions of E1 with ubiquitin and ATP as substrates and iodoacetamide (IAA), dithiothreitol (DTT) and pyrophosphatase as modifiers of the reaction constitute a complex metabolic pathway thoroughly explored by the groups of Ciechanover, Hershko, Rose and others $[4,5,6,7]$. The knowledge developed by these authors on this system is the cornerstone of the work shown below. We have recently approached the reactions catalyzed by E1 using ATP, $\mathrm{P}_{3}$ and $\mathrm{p}_{4} \mathrm{~A}$ as substrates and iodoacetamide and dithiothreitol as inhibitors of the reaction [3].

3) Two main processes can be considered in the ubiquitin proteasome system for degradation of proteins: a) ubiquitination of proteins and b) proteolytic cleavage of the tagged proteins by the $26 \mathrm{~S}$ proteasome complex. In the initial conjugative phase the ubiquitin activating en- 
zyme E1 catalyzes the formation of a high-energy thioester bond between the C-terminal of ubiquitin and a cysteine residue of E1; one of the several E2 enzymes (ubiquitin-carriers or ubiquitin conjugating enzymes) transfers the activated ubiquitin moiety from E1, via an additional high energy thioester intermediate, to the substrate that is specifically bound to a member of the ubiquitin-protein ligase family E3. The ubiquitinated protein is degraded by the proteasome with liberation of peptides and ubiquitin monomers $[7,8,9,10,11]$.

4) Finally, as shown below, we have applied our previous experience in the mathematical modeling of metabolic pathways of glucose [12], and purine nucleotide metabolism in rat brain [13] and Saccharomyces cerevisiae [14] to the ubiquitin proteasome system.

On the above bases, we have tried to develop a mathematical model of the reactions catalyzed by E1 in order to get new insights into the proteasome system in general, and on the role of E1 in this system. The description of the reactions catalyzed by E1 are presented (Figure 1) in a way that facilitates their mathematical writing with the powerful Mathematica Program [15]. The theoretical and experimental results can be compared, so that the analysis of some physiological aspects of E1, difficult to be explored in vivo or in vitro, can be attempted with the mathematical approach. In our view, the mathematical method here described may serve as a guide to explore other complex metabolic situations, changes in the concentration of intermediate enzyme forms of E1 (Figure 1), as well as to approach the mechanism of action of potential inhibitors of the enzyme. As an example of its predictive properties, the effect of AMP on the synthesis of $\mathrm{p}_{4} \mathrm{~A}$ by E1 was studied theoretically and confronted with the experimental results.

\section{EXPERIMENTAL PROCEDURES}

\subsection{Nomenclature}

Ubiquitin (abbreviated as $\mathrm{Ub}, \mathrm{U}, \mathrm{u}$ or $\underline{\mathrm{u}}$ ) is a small 76residue polypeptide containing a $\mathrm{C}$-terminal glycine, to which an adenylated residue can be covalently bound. The ubiquitin-activating enzyme will be named as E1, E or ${ }_{\text {нS }} \mathrm{E}$. The two functional regions or areas of this enzyme will be written at the left and at the right side of the letter E. The area at the left, corresponding to the U region (acronym of ubiquitin), may contain a molecule of ubiquitin covalently bound, by a thioester bond, to a cysteine residue of the enzyme (ubsE); this area is also called the ubiquitin donating area, as this ubiquitin can be donated to E2. The area at the right of E1 corresponds to the region named as A-u (acronym of AMP and ubiquitin), where the ubiquityl-adenylate (AMP-Ub) complex is tightly, but not covalently, bound to E1. The AMP-Ub complex is formed by the reaction between

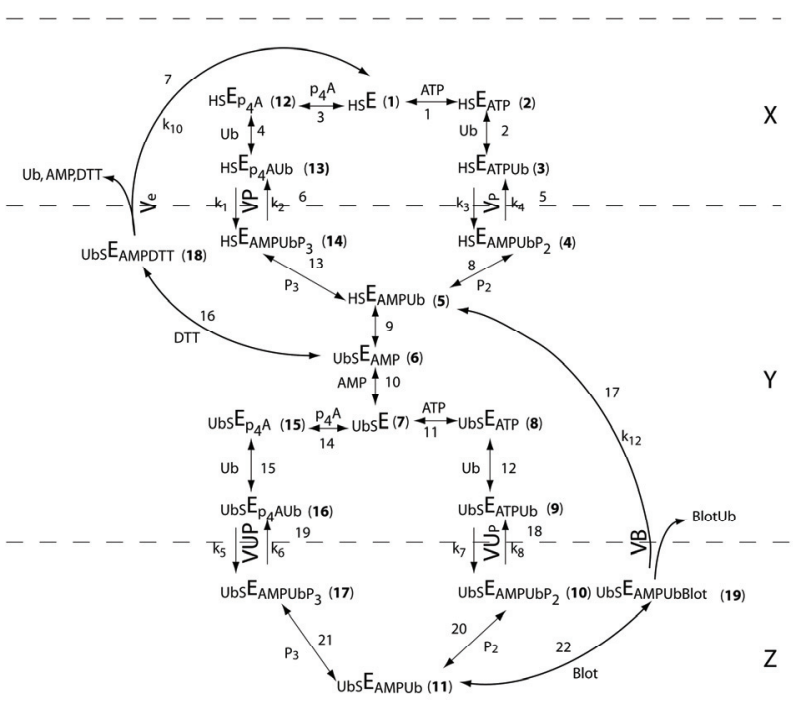

Figure 1. Scheme of the reactions considered for the ubiquitin activating enzyme E1. The following reactants were involved: ATP, adenosine 5'-tetraphosphate $\left(\mathrm{p}_{4} \mathrm{~A}\right)$, pyrophosphate $\left(\mathrm{P}_{2}\right)$, tripolyphosphate $\left(\mathrm{P}_{3}\right)$, ubiquitin (Ub), iodoacetamide (IAA), dithiothreitol (DTT), ubiquitin carrier-proteins (E2), ubiquitin protein-ligases (E3) and proteins to be ubiquitinated (E2 + E3 + proteins $=$ Blot in the Figure). The enzyme forms (EFs) of E1 with the potential reactants are also indicated with a number in bold face and between brackets. The reactions between two EFs have been indicated in regular face numbers from 1 to 22 . Three Boxes were contemplated: X, Y and Z. The reactions within each one of these Boxes are considered: in rapid equilibrium, reversible and indicated with two opposing arrow heads. The reactions between boxes are considered in steady state. For more details on nomenclature see the text.

one OH-residue of the phosphate moiety of AMP and the C-terminal of ubiquitin. The ubiquitin moiety of this complex is in an activated state, but it will not be donated to E2; the A-u area is also called the ubiquitin activating area. The different forms of the enzyme will be named as EF followed by a number in bold face. The reactions between two different EFs are designated with numbers from 1 to 22. The dissociation constants are named with two to five letters. The letters $\mathrm{x}, \mathrm{y}$ or $\mathrm{z}$ indicate that the reaction belongs to Box $\mathrm{X}, \mathrm{Y}$ or $\mathrm{Z}$, respectively (see below, Subsection 3.1.1); the other letters of the constant name indicate the substrate involved, as indicated between brackets: a (ATP); A ( $\left.\mathrm{p}_{4} \mathrm{~A}\right) ; \mathrm{u}, \mathrm{U}$ or $\underline{\mathrm{u}}$ (ubiquitin); $\mathrm{p}\left(\mathrm{P}_{2}\right) ; \mathrm{P}$ $\left(\mathrm{P}_{3}\right)$; e (AMP); d (DTT); B (Blot or the sum of E2 + E3 + Protein). The order of those letters indicates, in general, the sequence in which the reactants are bound to the enzyme complex. Forward reactions (Table 1, Appendix B) refer to those in the direction of the synthesis of EF 11, the enzyme form donating ubiquitin to E2; they have kinetic constants sub-indexed with uneven numbers; the contrary applies to the reverse reactions. 


\subsection{Computation}

The solving of the equations and the plotting of the solutions have been carried with a personal laptop computer Intel ${ }^{\circledR}$ Pentium ${ }^{\circledR}$ M processor $1.70 \mathrm{GHz}$ with the help of the Mathematica 5.0 Program [15].

\section{RESULTS AND DISCUSSION}

\subsection{The System}

The complete set of reactions catalyzed by E1, using ATP, $\mathrm{p}_{4} \mathrm{~A}, \mathrm{P}_{2}, \mathrm{P}_{3}, \mathrm{AMP}$, ubiquitin, and E2 as substrates and IAA and DTT as inhibitors are presented in Tables 1 and 2 and schematically shown in Figure 1. The reactions have been numbered from 1 to 22 . Those reactions identified with two opposing arrow heads indicate binding of substrates to E1 or rearrangement of substrates within the enzyme complex; they are considered in rapid equilibrium, following Michaelis-Menten postulates. The reactions noted with a simple arrow head, involving transformation of the bound substrate(s), are considered rate-limiting reactions and treated as being in steady state. The dissociation constants and the equilibrium constant (yue) are indicated in Table 1. The enzyme forms (EF) are expressed with the potential reactants or simply with a number in bold face, between brackets. As noted in Experimental procedures, the parts at the left and right side of $\mathrm{E} 1$ correspond to the $\mathrm{U}$ (acronym of ubiquitin) and to the A-u (acronym of AMP and ubiquitin) areas, respectively. The $\mathrm{U}$ area of $\mathrm{E} 1$ is the ubiquitin donating area to E2. The reactions depicted in Figure 1 can be dissected into two parts.

The one at the right side of Figure 1 represents the classical reactions previously described [7] using ATP, $\mathrm{P}_{2}$ and ubiquitin as substrates. In summary, ATP and ubiquitin are joined to E1 (reactions 1 and 2); the bound ATP is split into AMP $+P_{2}$ (reaction 5), the bound $P_{2}$ is liberated (reaction 8) and ubiquitin is displaced from the A-u to the $U$ region of the enzyme, with concomitant liberation of AMP (reactions 9 and 10). Once ubiquitin is covalently joined to the $\mathrm{U}$ region of $\mathrm{E}$ 1, another moiety of ubiquitin is bound to the A-u area of the enzyme (using ATP as co-substrate) through a sequence of reactions (reactions 11, 12, 18, 20) similar to those previously described (reactions 1, 2, 5, 8). The enzyme form (EF 11) is already prepared to serve as donor of ubiquitin from the U area of E1 to E2 (named as "Blot" in Figure 1). The EF 9 generated in this step is ready to repeat the cycle, catalyzed by reactions $9-12,18$, and 20 and to afford the ubiquitin molecules needed for the processing of the proteins by the proteasome.

The part at the left side of Figure 1 shows similar reactions to those just described with the exception that $\mathrm{p}_{4} \mathrm{~A}$ serves as a source of energy instead of ATP (compare reactions 1 and 11 with reactions 3, 14, respectively), and a molecule of $\mathrm{P}_{3}$, instead of $\mathrm{P}_{2}$, is involved in
Table 1. Reactions in rapid equilibrium inside boxes $\mathrm{X}$, Y, and Z. (Figure 1). The number, substrates and product of the reactions are as indicated in the Table and in Figure 1. The dissociation (equilibrium) constants were named according to the following rules: the binding of a reactant to the A-u area of E1 is represented with a letter between brackets: $\mathrm{P}_{2}(\mathrm{p}), \mathrm{P}_{3}(\mathrm{P})$, ATP (a), $\mathrm{p}_{4} \mathrm{~A}(\mathrm{~A}), \mathrm{Ub}(\mathrm{u}), \operatorname{AMP}(\mathrm{e}), \operatorname{DTT}(\mathrm{d})$ or Blot (B); x, y or z indicates their location in boxes $\mathrm{X}, \mathrm{Y}$ or $\mathrm{Z}$; $\underline{\mathrm{u}}$ represents the oscillation of ubiquitin from the A-u to the $\mathrm{U}$ area of E1; $U$ represents ubiquitin bound to a-SH group of the $\mathrm{U}$ area of E1. For more details on the nomenclature of the constants see Experimental procedures. Dissociation constants are defined as the product of the concentration of the substrates divided by the concentration of the bound complex (product $\mathrm{EF}$ ); for example, constant $\mathrm{xa}={ }_{\mathrm{HS}} \mathrm{E} \times \mathrm{ATP} / \mathrm{HS}_{\mathrm{ATP}}$. The equilibrium constant yue is defined as ${ }_{\mathrm{UbS}} \mathrm{E}_{\mathrm{AMP}} / \mathrm{HS}_{\mathrm{HS}} \mathrm{E}_{\mathrm{AMPUb}}$. Numeric values for these constants have either been taken from the literature or tuned $(*)$ to reflect available experimental data. To facilitate location of the reactions in Figure 1, EFs are also indicated by their assigned numbers (in bold characters between brackets).

\begin{tabular}{|c|c|c|c|}
\hline & \multicolumn{2}{|r|}{ Reaction } & \multirow{2}{*}{$\begin{array}{l}\text { Constant } \\
\text { value }(\mu \mathrm{M})\end{array}$} \\
\hline & Number & Substrates, dissociation constant, product & \\
\hline \multirow{4}{*}{ 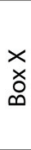 } & 1 & ${ }_{H S} E(1)+A T P \stackrel{x a}{\longrightarrow} H_{S} E_{A T P}(2)$ & (ATP) $4.9[8,23]$ \\
\hline & 2 & ${ }_{H S} \mathrm{E}(1)+\mathrm{p}_{4} \mathrm{~A} \stackrel{\mathrm{XA}}{\longrightarrow} \mathrm{HSE}_{\mathrm{p} 4 \mathrm{~A}}(\mathbf{1 2})$ & $\left(p_{4} A\right) 150.0^{\#}$ \\
\hline & 3 & $\mathrm{HS}_{\mathrm{ATP}}(\mathbf{2})+\mathrm{Ub} \stackrel{\mathrm{xau}}{\longrightarrow} \mathrm{HS}_{\mathrm{ATPUb}}(\mathbf{3})$ & (Ub) $2.0[8,23]$ \\
\hline & 4 & ${ }_{H S} E_{p 4 A}(12)+U b \stackrel{X A u}{\longrightarrow} H S E_{p 4 A U b}(13)$ & (Ub) $2.0[8,23]$ \\
\hline \multirow{9}{*}{ ટ㐅㐅̆ } & 8 & $\mathrm{HS}_{\mathrm{S}} \mathrm{E}_{\mathrm{AMPUb}}(\mathbf{5})+\mathrm{P}_{2} \stackrel{\text { yuep }}{\longrightarrow} \mathrm{HS} \mathrm{E}_{\text {AMPUbP2 }}$ (4) & $\left(P_{2}\right) 550^{\#}$ \\
\hline & 13 & $\mathrm{HS}_{\mathrm{AMPUb}}(\mathbf{5})+\mathrm{P}_{3} \stackrel{\text { yueP }}{\longrightarrow} \mathrm{HS}_{\mathrm{AMPUbP} 3}(\mathbf{1 4})$ & $\left(P_{3}\right) 2800[8]$ \\
\hline & 9 & ${ }_{\mathrm{HS}} \mathrm{E}_{\mathrm{AMPUb}}(\mathbf{5}) \stackrel{\text { yue }}{\longrightarrow} \mathrm{Ubs} \mathrm{E}_{\mathrm{AMP}}(\mathbf{6})$ & $2.0[5]$ \\
\hline & 10 & $\mathrm{Ubs} \mathrm{E}(\mathbf{7})+\mathrm{AMP} \stackrel{\text { Uye }}{\longleftrightarrow} \mathrm{Ubs}_{\mathrm{AMP}} \mathrm{E}_{\mathrm{AM}}(\mathbf{6})$ & (AMP) $5[5]$ \\
\hline & 16 & $\mathrm{UbS}_{\mathrm{AMP}}(\mathbf{6})+\mathrm{DTT} \stackrel{\text { Uyed }}{\longrightarrow} \mathrm{Ubs} \mathrm{E}_{\mathrm{AMPDTT}}(\mathbf{1 8})$ & $2250^{\#}$ \\
\hline & 11 & $U b s E(7)+A T P \stackrel{\text { Uya }}{\longrightarrow} U b s E_{A T P}(8)$ & (ATP) $4.9[8,23]$ \\
\hline & 14 & $U b s=(7)+p_{4} A \stackrel{U y A}{\longrightarrow} U b s s_{p 4 A}(15)$ & (p4A) $150.0^{\#}$ \\
\hline & 12 & $U_{b s} E_{A T P}(8)+U b \stackrel{\text { Uyau }}{\longrightarrow}$ Ubs $E_{A T P U b}(9)$ & (Ub) $2.0[8,23]$ \\
\hline & 15 & $U b s E_{p 4 A}(15)+U b \stackrel{U y A u}{\longrightarrow} U b s E_{p 4 A U b}(16)$ & (Ub) $2.0[8,23]$ \\
\hline \multirow{3}{*}{$\begin{array}{l}N \\
\times \\
\stackrel{\infty}{0}\end{array}$} & 20 & Ubs $E_{\text {AMPUb }}(11)+P_{2} \stackrel{\text { Uzuep }}{\longrightarrow}$ Ubs $_{\text {AMPUbP2 }}(10)$ & (P2) $550^{\#}$ \\
\hline & 21 & $\mathrm{Ubs}_{\mathrm{amPUb}}(11)+\mathrm{P}_{3} \stackrel{\text { UzueP }}{\longrightarrow} \mathrm{UbS}_{\mathrm{A}} \mathrm{E}_{\mathrm{AMPUbP} 3}(17)$ & (P3) $2800[8]$ \\
\hline & 22 & Ubs $E_{\text {AMPUb }}(11)+B l o t \stackrel{\text { UzueB }}{\longrightarrow}$ Ubs $E_{\text {AMPUbBlot }}$ (19) & $33000^{\#}$ \\
\hline
\end{tabular}

Table 2. Rate constant values (simplified procedure) of the steady state reactions. The constants with uneven or even numbers indicate forward or reverse direction, respectively, towards formation of ${ }_{\mathrm{UbS}} \mathrm{E}_{\mathrm{AMPUb}}$ (EF 11, the enzyme complex donating $\mathrm{Ub}$ to the ubiquitin carrier proteins $\mathrm{E} 2$ ).

\begin{tabular}{cccc}
\hline Constants & Reactions & Value $\left(\mathrm{s}^{-1}\right)$ & References \\
\hline $\mathrm{k} 1=\mathrm{k} 3$ & Vp; VP & $0.75 \times \mathrm{k} 5$ & {$[4]$} \\
$\mathrm{k} 2=\mathrm{k} 4$ & Vp; VP & $0.75 \times \mathrm{k} 6$ & {$[4]$} \\
$\mathrm{k} 5=\mathrm{k} 7$ & VUp; VUP & 0.13 & Tuned \\
$\mathrm{k} 6=\mathrm{k} 8$ & VUp; VUP & $6.25 \times \mathrm{k} 5$ & {$[4]$} \\
$\mathrm{k} 10$ & Ve & 0.57 & Tuned \\
$\mathrm{k} 12$ & VB & 3.3 & Tuned \\
\hline
\end{tabular}

the process (compare reactions 8 and 20 with reactions 13 and 21, respectively). Treatment of this part of Figure 1 is based on previous publications from our laboratory [3], 
with the assumption that the binding of $\mathrm{p}_{4} \mathrm{~A}$ and $\mathrm{P}_{3}$, behave similarly to the binding of ATP and $\mathrm{P}_{2}$, respectively.

\subsubsection{Kinetics Considerations}

The kinetic treatment followed here is based on the general consensus on how to handle rapid equilibrium and steady state reactions of uni-reactant enzymes [16] and on the simplifications introduced by Cha [17]. He showed that the calculation of rates of equations of complex enzyme systems could be simplified by treating all the enzyme forms, implying binding of a substrate to an enzyme within a rapid equilibrium segment, as a single entity. On the contrary, reactions involving transformation of the previously bound substrates are considered rate-limiting reactions and treated as being in a steady state.

Following these criteria three set of reactions, considered in rapid equilibrium among themselves, were arranged in Boxes X, Y and Z. Reactions 1-4, in which binding of ATP, $\mathrm{p}_{4} \mathrm{~A}$, or $\mathrm{Ub}$, to $\mathrm{E} 1$ take place, were grouped in Box X; Reactions 8-12 and 14-15, implying the binding of $\mathrm{P}_{2}, \mathrm{P}_{3}$, AMP, ATP, $\mathrm{p}_{4} \mathrm{~A}$ and ubiquitin to E1, were grouped in Box Y. Reactions 20-22, catalyzing binding of $\mathrm{P}_{2}$, or $\mathrm{P}_{3}$ to $\mathrm{E} 1$ and transfer of ubiquitin from E1 to E2 were grouped in Box Z.

The reactions considered as being in steady state were those implying cleavage of ATP (reactions 5 and 18) or of $p_{4} A$ (reactions 6 and 19) (Figure 1). These reactions interconnect Boxes X-Y and Boxes Y-Z).

\subsubsection{Reactions in the Presence of Added Dithiothreitol (DTT), Iodoacetamide (IAA), Blot or Pyrophosphatase}

As shown by Haas [6], DTT does not split the ubiquityl adenylate complex if it is tightly bound to the A-u area of E1 (as in EF 5). However, DTT breaks the thioester bond between $\mathrm{Ub}$ and a cysteine of E1 (Figure 1, EF 6), with formation of DTT-Ub. This last complex is rather unstable due either to the ability of ubiquitin itself to monitor its own C-terminal for the occurrence of thioester bond or to a possible contaminant thiolesterase present in the sample of E1 [6]. Iodoacetamide (IAA) binds covalently to -SH group(s) here indicated (schematically) at the left side of all the EFs (Figure 1). Particularly important, in the context of this work, is the reaction between IAA and the -SH residue of EF 5, to which ubiquitin is linked prior to its transference to E2. The effect of IAA (as it blocks reaction 9) results in a displacement of all the enzyme forms (EFs) of Figure 1 towards those above EF 5, with the subsequent disappearance of EFs 6-11 and 15-17.

With respect to the effect of E2 on E1, E1 can also be viewed as the first enzymatic step for the ubiquitination of proteins and further degradation by the proteasome. To simulate the whole ubiquitination process a hypothetical reactant named Blot (from Blotting of ubiquitin) has been introduced. Blot is the sum of ubiquitin carrier proteins (E2), ubiquitin protein ligases (E3) and protein(s) to be ubiquitinated, and represents the destiny for all the ubiquitin molecules activated by E1. To simulate physiological conditions, Blot is supposed to be in large excess over E1. The reaction between EF 11 and the entity Blot has been named 22 and was considered in rapid equilibrium (Figure 1).

Finally, the effect of the addition of PPase to the system has been easily simulated by the following reaction, assumed to have Michaelis-Menten kinetics:

$$
\text { PPase }+\mathrm{P}_{2}<\longrightarrow \text { PPase }_{2} \longrightarrow \text { PPase }+2 \mathrm{P}_{\mathrm{i}}
$$

Thus, PPase hydrolyzing the $\mathrm{P}_{2}$ liberated in reaction 8 (between EFs 4-5) and 20 (between EFs 10-11) displaces the equilibrium towards EF 5 and EF 11, respectively (Figure 1).

\subsection{Mathematical Model}

The mathematical model, a symbolic expression of the enzyme reactions assumed above (Figure 1 and Tables 1 and 2), was developed with the help of the Mathematica Program according to the following steps:

\subsubsection{Application to the Rapid Equilibrium \\ Reactions Inside Boxes $X, Y$ and $Z$ \\ (Supplemental Data, Appendix A)}

The concentrations of the enzyme forms in rapid equilibrium were calculated following a similar procedure in each one of the Boxes: 1) each dissociation constant was equalled to its corresponding value according to the substrates and products involved; 2) the sum of the EFs contained in Boxes $\mathrm{X}, \mathrm{Y}$ and $\mathrm{Z}$ were equalled to $\mathrm{X}, \mathrm{Y}$ and $Z$, respectively; 3) the values of the 19 EFs to be calculated (5 in Box X; 10 in Box Y and 4 in Box Z) were requested with the command "Solve" of the Mathematica Program (Supplemental data, Appendix A). The rather cumbersome resulting equations, were disentangled with the command "Simplify" and used in the following step.

\subsubsection{Steady State Reactions between Boxes $X-Y$ and Y-Z (Supplemental Data, Appendix B)}

The resulting equations for the $10 \mathrm{EFs}$ involved in the steady state reactions were used to determine the actual net velocities: Vp (between EFs 3 and 4); VP (between EFs 13 and 14); VUp (between EFs 9 and 10); VUP (between EFs 16 and 17); Ve (between EF 18 and 1) and VB (between EFs 19 and 5). The velocities Ve and VB were also included to cope with situations described below, where the presence of dithiothreitol and E2 are contemplated. Those six velocities (Vp, VP, VUp, VUP, $\mathrm{Ve}$ and VB), were calculated as functions of the concentrations of EFs (as determined in Appendix A), the rate constants (k1-k8, k10, k12), and taking into account the forward (towards the synthesis of EF 11) and the reverse reactions (Supplemental data, Appendix B, a). In part b of Appendix B, the values of the 19 enzyme forms cal- 
culated in Appendix A are presented; note that these values are grouped into two sections: 1) comprising those values directly involved in the steady state situation, and 2) the values implicit in the anterior formulas and needed for their solutions. In part c of Appendix B the statements needed for the solution of the equations are given: the total enzyme is defined as the sum of the amount of enzyme in Boxes X, Y and Z; by application of the steady state treatment suggested by Cha to the diagram of reactions (Figure 1), the net flux between boxes $\mathrm{X}$ and $\mathrm{Y}(\mathrm{VP}+\mathrm{Vp}-\mathrm{Ve})$ and between boxes $\mathrm{Y}$ and $\mathrm{Z}$ (VUP + VUp-VB) is set equal to zero and, finally, the unknowns to be solved are listed.

\subsubsection{Ordinary Differential Equations System (Supplemental Data, Appendix C)}

The changes in the concentration of reactants (or even of EFs) with the incubation time can be calculated with a system of differential equations performed with the Command "NDSolve" of the Mathematica Program, once the expression of the velocities are known. The syntax for this procedure admits many variants depending on the entity to be clarified. As an example, changes in the concentrations of ATP, $\mathrm{p}_{4} \mathrm{~A}, \mathrm{P}_{3}, \mathrm{P}_{2}$, AMP, Blot or BlotUb are presented in Supplemental data, Appendix C. The Program admits other requested variants. The parameter values, initial assay conditions, and time of the reaction must also be included. Concerning the parameter values, two Procedures have been employed: Comprehensive and Simplified. The Comprehensive Procedure allows the theoretical calculation of all the variables of the system (19 EFs) as a function of: a) the total amount of E1, b) the value of the 15 dissociation constants and of the equilibrium constant yue, c) the value of the 10 rate constants, and d) the concentration of the reactants. This can be contemplated as an ideal scenery, helpful for example, to handle reactions that can not be performed experimentally, to investigate the role of each constant on the system and to calculate the concentrations of EFs and of the reactants under different experimental conditions, etc. However, given the scarcity of available experimental data, we considered it more convenient to introduce the Simplified Procedure (Table 2) with the following assumptions: the dissociation constant values for the binding of ATP, $\mathrm{p}_{4} \mathrm{~A}, \mathrm{P}_{2}, \mathrm{P}_{3}, \mathrm{Ub}$, AMP to any EF were set equal for each substrate, irrespective of the enzyme form (EFs) to which they bind (Figure 1, Tables 1 and 2); the values of the dissociation constants were taken from the literature, calculated as the average of different reported values (when this was the case) or tuned to fit the experimental results when no data on a particular constant had been reported (Tables 1 and 2); the constant UzueB (dissociation constant between EF 11 and EF 19 (Figure 1) was chosen arbitrarily as equal to $33 \mathrm{mM}$, since Blot is an hypothetical substrate that represents the sum of E2 + E3 + proteins (theoretically considered in large excess over E1); ii) the rate constants of each one of the pairs $(\mathrm{k} 1, \mathrm{k} 3),(\mathrm{k} 2, \mathrm{k} 4)$, $(\mathrm{k} 5, \mathrm{k} 7)$ and $(\mathrm{k} 6, \mathrm{k} 8)$ were given the same value, as they correspond to similar catalyzed reactions (Table 2 ); from some experimental data $[3,4]$ it can be deduced that the values of $(\mathrm{k} 1, \mathrm{k} 3)$ and $(\mathrm{k} 2, \mathrm{k} 4)$ are $75 \%$ of those of $(\mathrm{k} 5$, k7) and (k6, k8), respectively (Table 1 and 2) (Figure 1); finally, a previous study [4] specifically calculated that $\mathrm{k} 7 / \mathrm{k} 8=0.16$; rate constant $\mathrm{k} 5$ was tuned to $0.13 \mathrm{~s}^{-1}$; rate constant k12 was arbitrarily made equal to $3.3 \mathrm{~s}^{-1}$, as we only wanted to compare the effect of different assay conditions in the ubiquitination rate of Blot. All these data are sufficient to fix one of the eight rate constants, allowing the rest of the seven constants to be calculated automatically by the program

\subsection{Experimental Test of the Mathematical Model}

Once the mathematical model was established and simplified, it was critical to confront it with the experimental results. As the mathematical scanning of the plethora of experimental results available on E1 would be beyond the scope of the present study, only the reactions taking place in the presence of ubiquitin, ATP, $\mathrm{p}_{4} \mathrm{~A}$ and $\mathrm{P}_{3}$ and the influence of DTT, IAA, pyrophosphatase and Blot $(\mathrm{E} 2+\mathrm{E} 3+$ proteins $)$ on these reactions are considered. The advantage of $\mathrm{P}_{3}$ over $\mathrm{P}_{2}$ concerning the evaluation of the experimental results should be stressed. Whereas in the presence of ATP $\left( \pm \mathrm{P}_{2}\right)$ synthesis or degradation of ATP is observed [5] (i.e. no new compound is synthesized and the reaction could be studied mainly using radioactive substrates), in the presence of $\mathrm{ATP}\left( \pm \mathrm{P}_{3}\right)$ the rate of synthesis of a new compound $\left(\mathrm{p}_{4} \mathrm{~A}\right)$ can be used as a tool to follow the course of the reaction.

\subsubsection{Effect of Dithiothreitol (DTT)}

According to the experimental findings reported by Haas [6], the effect of DTT has been here mathematically reproduced through the rapid equilibrium binding of DTT to EF 6, consequent breakage of the thioester bond between $\mathrm{Ub}$ and a cysteine located in the $\mathrm{U}$ region of the enzyme, formation of EF 1 and final liberation of AMP, DTT and Ub to the medium (Figure 1). The effect of DTT is theoretically clear: the synthesis of AMP from ATP is produced and AMP is liberated irreversibly to the reaction medium with displacement of the reactions toward the enzyme forms located above EF 6 in the scheme shown in Figure 1; the synthesis of $\mathrm{p}_{4} \mathrm{~A}$ takes place both through reaction 3, between EF 12 and EF 1 (Box X), and through reaction 14, between EF 15 and EF 7 (Box Y). As a consequence of the disappearance of the enzyme forms located below EF 6 by the effect of DTT, changes in the rate of synthesis of $\mathrm{p}_{4} \mathrm{~A}$ in the presence of DTT would be indicative of the relative rate of synthesis of $\mathrm{p}_{4} \mathrm{~A}$ by reaction 6 (between Boxes X-Y) 
versus reaction 19 (between Boxes Y-Z). The experimental and theoretical rates of syntheses of AMP and $\mathrm{p}_{4} \mathrm{~A}$ (from ATP and $\mathrm{P}_{3}$ ), in the absence of DTT or in the presence of increasing concentrations of DTT, are represented in Figure 2. The rate of synthesis of AMP clearly increased in the presence of DTT whereas the rate of synthesis of $\mathrm{p}_{4} \mathrm{~A}$ decreased slightly (about $8 \%$ ) [3], indicating that the synthesis of $\mathrm{p}_{4} \mathrm{~A}$ takes place (in the absence of DTT) preferentially by reaction 3 (between EF 12 and EF 1). The experimental and the theoretical results agree quite well. The strict linearity of the experimental approach (in the upper panels of Figure 2 reflects the fact that only one experimental point was considered. The theoretical maximum velocities were always calculated from the information provided by the suppliers on the amount of protein, enzyme activity and molecular weight of E1. Concerning the synthesis of $\mathrm{p}_{4} \mathrm{~A}$ (panels at the right), the apparent discrepancies between the experimental and theoretical results are attributable to the amount of active enzyme actually present in that assay. The effect of DTT on the ubiquitination of E2 (Blot) will be presented further on.

\subsubsection{Effect of Iodoacetamide (IAA)}

The experimental results (Figure 3 ) obtained with IAA

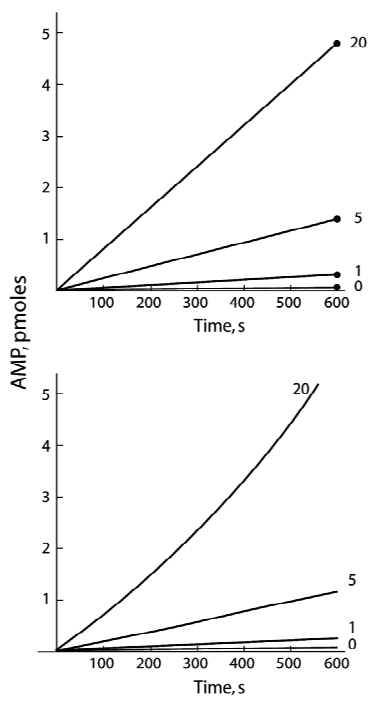

(a)

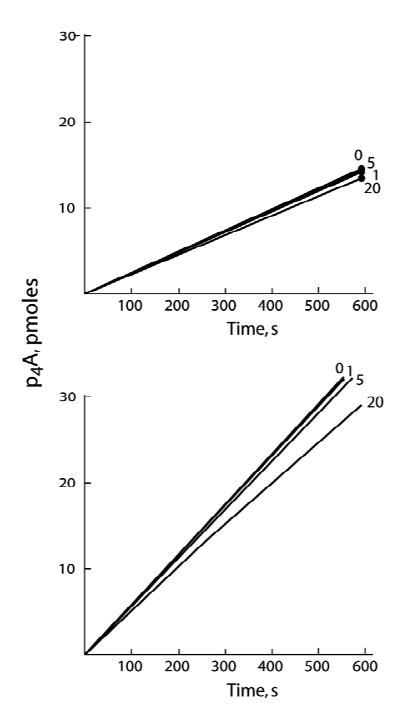

(b)
Figure 2. Influence of dithiothreitol (DTT) on the synthesis of AMP (panels on the left, A) and on the synthesis of $\mathrm{p}_{4} \mathrm{~A}$ (panels on the right, B): experimental results (upper panels) and theoretical calculation (lower panels). Initial assay conditions: 0.02 mM ATP; $6 \mu \mathrm{M}$ ubiquitin; $0.8 \mathrm{mM} \mathrm{P}_{3} ; 0.05 \mu \mathrm{g}$ PPase; $0 ; 1 ; 5$ and $20 \mathrm{mM}$ DTT as indicated, and $1 \mathrm{pmol}$ of E1. Time of the reaction $600 \mathrm{~s}$. Experimental data were taken from [3] and theoretical calculations were obtained with the Mathematica Program as indicated in the text. Note that the synthesis of AMP is undetectable in the absence of DTT, both in experimental and theoretical conditions.

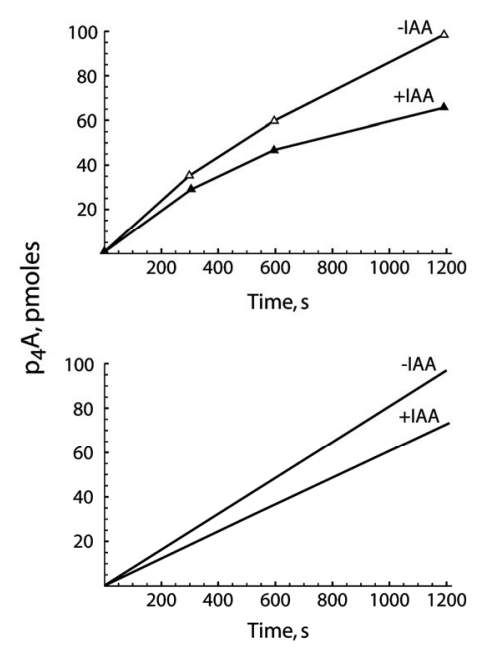

Figure 3. Influence of iodoacetamide (IAA) on the synthesis of $\mathrm{p}_{4} \mathrm{~A}$ and (not represented) on the synthesis of AMP: experimental and theoretical results. E1 at a final concentration of $0.75 \mathrm{nmol} / \mathrm{ml}(1.5$ pmol of E1) was incubated in the presence of: 50 $\mathrm{mM}$ Tris- $\mathrm{HCl}, \mathrm{pH} 7.5$, and $1 \mathrm{mg} / \mathrm{ml}$ BSA for 30 $\min$ at $37^{\circ} \mathrm{C}$ and in the absence or presence of 0.5 mM IAA. Initial assay conditions were $0.02 \mathrm{mM}$ ATP; $6 \mu \mathrm{M}$ ubiquitin; $0.8 \mathrm{mM} \mathrm{P}_{3}$; $0.05 \mu \mathrm{g}$ PPase. DTT was omitted. Time of the reaction was $1200 \mathrm{~s}$. Experimental data (upper panel) are taken from [3]. Theoretical results (lower panel) were obtained with the Mathematica Program. Synthesis of AMP was undetectable by both procedures and therefore is not represented in the Figure.

were performed with E1 previously incubated with IAA [5]. According to [4,5], IAA reacts covalently with a critical $-\mathrm{SH}$ residue located in the $\mathrm{U}$ region of $\mathrm{E} 1$, blocking its reaction with ubiquitin. This inhibition was simulated mathematically by giving the value zero to the equilibrium constant yue (Figure 1, reaction 9, between EF 5 and EF 6). Although the effect of IAA and DTT on E1 may seem apparently similar (both split the scheme of reactions presented in Figure 1 into two parts, above and below a specific enzyme form: EF 5 and EF 6, respectively), the consequences are drastically different concerning the synthesis of AMP. Whereas DTT stimulates, IAA completely inhibits the synthesis of AMP, as EF 6 is not formed in the presence of IAA (see Figure 3 and its legend). Following a similar reasoning as above, the effect of DTT and IAA on the synthesis of $\mathrm{p}_{4} \mathrm{~A}$ could be expected to be inhibitory. The experimental results pointed in that direction, with IAA being a more potentinhibitor of the synthesis of $\mathrm{p}_{4} \mathrm{~A}(25 \%$, Figure 3$)$ than DTT (8\%, Figure 2) [3].

\subsubsection{Influence of Pyrophosphatase}

In the presence of $\mathrm{Ub}, \mathrm{ATP}$, and $\mathrm{P}_{3}$, synthesis of $\mathrm{P}_{2}$ takes place (Figure 1) in reactions 5 and 8 (with the participa- 
tion of EFs 3-5) and in reactions 18 and 20 (with participation of EFs 9-11); $\mathrm{P}_{2}$ is a substrate for the synthesis of ATP in the same reactions as above, acting in the reverse direction. In the presence of PPase, $\mathrm{P}_{2}$ is degraded and reactions 8 and 20 cannot proceed in the reverse direction (Figure 4). Consequently, in the presence of PPase and $\mathrm{P}_{3}$ the synthesis of $\mathrm{p}_{4} \mathrm{~A}$ is favored, by displacement of the E1 cycle towards the left part of Figure 1, i.e. toward the synthesis of $\mathrm{p}_{4} \mathrm{~A}$. Previous results from this laboratory [3] had shown that the addition of PPase to a reaction mixture containing $\mathrm{P}_{3}$, almost doubled the rate of synthesis of $\mathrm{p}_{4} \mathrm{~A}$ and slightly inhibited the synthesis of AMP. The theoretical and experimental results on the influence of PPase on the synthesis of AMP and $\mathrm{p}_{4} \mathrm{~A}$ are shown in Figure 4.

\subsection{4. $\mathbf{p}_{4} \mathrm{~A}$ as Substrate of the Reaction}

This experiment was performed by allowing a complete transformation of $\left[\mathrm{a}^{32} \mathrm{P}\right] \mathrm{ATP}$ into labeled $\left[\mathrm{a}-{ }^{32} \mathrm{P}\right] \mathrm{p}_{4} \mathrm{~A}$ in the presence of $\mathrm{Ub}, \mathrm{P}_{3}$ and PPase [3]. Further incubation of this reaction mixture converted gradually the synthesized $\mathrm{p}_{4} \mathrm{~A}$ into AMP thanks to the effect of DTT. The experimental and theoretical results are shown in Figure 5, demonstrating that $\mathrm{p}_{4} \mathrm{~A}$ is a substrate for the synthesis of AMP through EFs 12-14, 5, 6, 18 and 1. In this way, when the final equilibrium is reached, AMP is the only destiny for all the ATP initially present in the assay mixture. The small differences between the experimental and theoretical results might be related to the inactivation of the enzyme after prolonged times of incubation (Figure 5).

\subsubsection{Influence of $E 2$}

In the presence of only ATP and ubiquitin, the enzyme catalyzes a rapid pre-steady state situation where only two equivalents of ATP per equivalent of enzyme are hydrolyzed [4]. The hydrolysis of ATP can be stimulated by the addition of $\mathrm{P}_{3}$ (as in previous sections) or by the addition of an ubiquitin acceptor (Figure 1). This last situation is here simulated by the addition of Blot, a mixture of E2 + E3 + proteins, representing a sink for the activated ubiquitin. The main reaction in the presence of Blot is represented by:

$$
{ }_{\mathrm{UbS}} \mathrm{E}_{\mathrm{AMPUb}}(\mathrm{EF} 11)+\text { Blot }<{ }_{\mathrm{UbS}} \mathrm{E}_{\mathrm{AMPUbBlot}}(\mathrm{EF} \text { 19) }
$$

$\rightarrow{ }_{\mathrm{HS}} \mathrm{E}_{\mathrm{AMPUb}}(\mathrm{EF}$ 5)+ BlotUb

These steps have been simulated by one reversible reaction in rapid equilibrium (reaction 22 between EF 11 and EF 19) and one reaction in steady state (VB). This last reaction (17) is considered irreversible because of the physiological large excess of (E2 + E3 + proteins) over E1 (Figure 1). The complete cycle for the hydrolysis of ATP in the presence of Blot is composed of reactions 22, 17, 9-12, 18, 20 (Figure 1). The influence of Blot (at $0,0.5,1,2,5,15 \mathrm{mM}$ ) on the reaction catalyzed

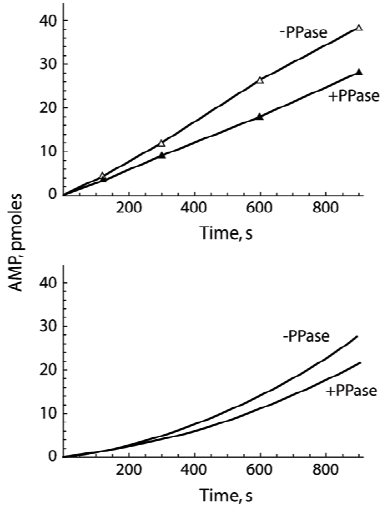

(a)

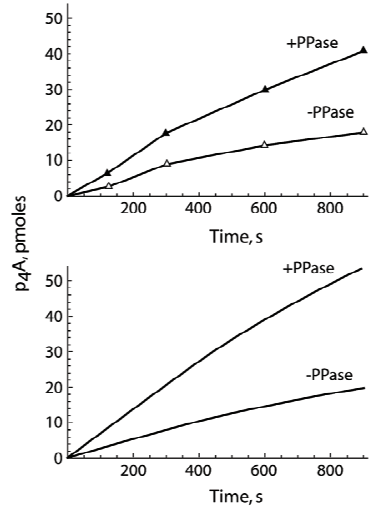

(b)
Figure 4. Influence of PPase on the synthesis of AMP (left-hand panels $\mathrm{A}$ ) and $\mathrm{p}_{4} \mathrm{~A}$ (right-hand panels, B): experimental and theoretical results. Initial assay conditions: 0.02 mM ATP; $6 \mu \mathrm{M} \mathrm{Ub} ; 0.8 \mathrm{mM} \mathrm{P}_{3} ; 20 \mathrm{mM}$ DTT; 1.5 pmol E1 and, when indicated, $0.05 \mu \mathrm{g}$ PPase. Reaction time: $900 \mathrm{~s}$. Experimental data were taken from [3] and represented in the upper part of both panels. The theoretical results are represented in the lower panels.

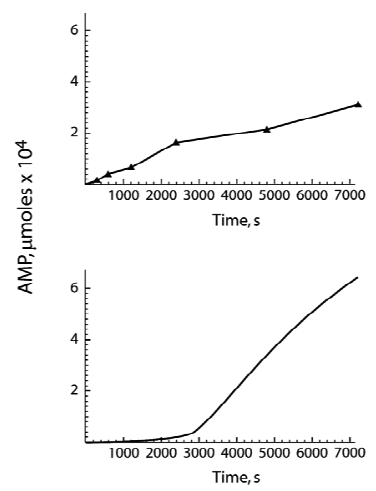

(a)

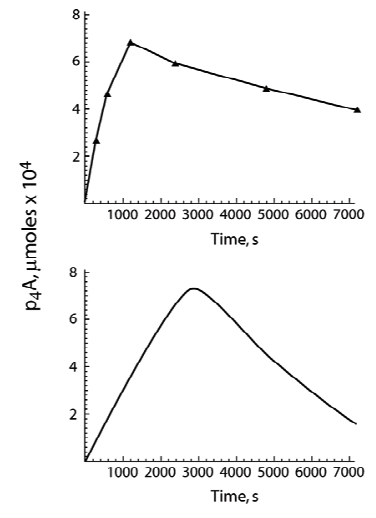

(b)
Figure 5. Adenosine 5'-tetraphosphate $\left(\mathrm{p}_{4} \mathrm{~A}\right)$ as substrate for the synthesis of AMP: experimental and theoretical results. Initial assay conditions: $0.04 \mathrm{mM}$ ATP; $12 \mu \mathrm{M} \mathrm{Ub} ; 0.8 \mathrm{mM} \mathrm{P}_{3}$; $0.05 \mu \mathrm{g}$ PPase; $2 \mathrm{mM}$ DTT and $4 \mathrm{pmol}$ E1. The reaction time was increased to $7200 \mathrm{~s}$ ( 2 hours) in order to consume all the ATP in the assay. In this way, the synthesis and posterior degradation of $\mathrm{p}_{4} \mathrm{~A}$ towards formation of AMP can be observed, as a function of incubation time. Experimental data were taken from [3] and represented in the upper part of both panels. Theoretical results obtained for the synthesis of AMP and $\mathrm{p}_{4} \mathrm{~A}$ are represented in the lower panels.

by E1 is represented in Figure 6 panels a and b. In the absence of Blot changes in the concentration of ATP, or synthesis of AMP (not represented) are undetectable (Figure 6 (a)); the rate of disappearance of ATP increased with increasing concentrations of Blot, with an apparent activation constant of about $1.5 \mathrm{mM}$ for Blot in 


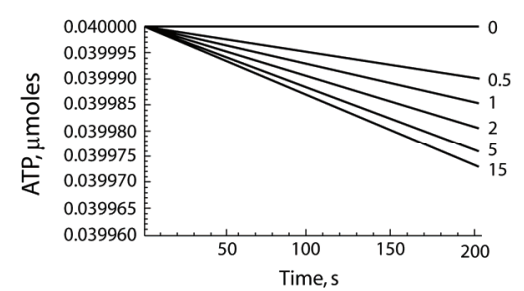

(a)

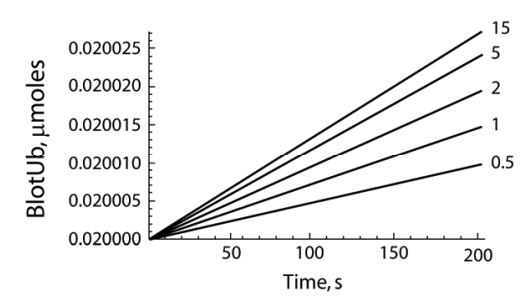

(b)

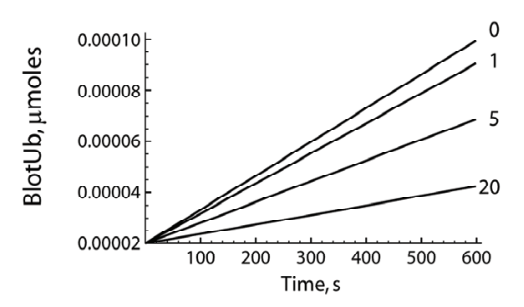

(c)

Figure 6. Theoretical approach for ubiquitination of Blot (E2+E3+proteins) by E1 and inhibition of this process by DTT. The pre-established theoretical conditions in the upper panels were: $2 \mathrm{mM}$ ATP; $6 \mu \mathrm{M} \mathrm{Ub}$; 1.5 pmol E1; $0.05 \mu \mathrm{g}$ PPase and Blot $(0 ; 0.5 ; 1 ; 2 ; 5$ and $15 \mathrm{mM}$ ). Time of reaction: $200 \mathrm{~s}$. In the absence of Blot and DTT, the concentrations of ATP remain constant. Upon addition of increasing concentrations of the Blot reactant (which represents a sink for activated ubiquitin) consumption of ATP (panel a) and formation of ubiquitinated Blot (BlotUb) (panel b) can be observed. In panel c, the pre-established theoretical conditions are as above, except that AMP $(0.8 \mathrm{mM})$ and Blot $(65 \mathrm{mM})$, were included to simulate physiological conditions; DTT was included at theoretical concentrations of $0,1,5$ and $20 \mathrm{mM}$ as indicated. Time of reaction: $600 \mathrm{~s}$.

these theoretical conditions (Figure 6 (a)). The influence of the concentration of Blot on the synthesis of BlotUb followed a similar pattern to that observed for the degradation of ATP (Figure 6 (b)). Finally, the influence of DTT on the ubiquitination process was tested in the presence of physiological concentrations of ATP $(2 \mathrm{mM})$, AMP $(0.8 \mathrm{mM})$ and Blot $(\mathrm{E} 2+\mathrm{E} 3+$ protein $)(65 \mathrm{mM})$. Inhibition rates for the ubiquitination of Blot of 10,32 and $57 \%$ were calculated in the presence of 1,5 and 20
mM DTT (Figure 6 (c)). This inhibition by DTT was due to the displacement of the equilibrium of the system away from $_{\mathrm{UbS}} \mathrm{E}_{\mathrm{AMPUb}}$ (EF 11), the enzyme form donating $\mathrm{Ub}$ to $\mathrm{E} 2$.

\subsubsection{Responsiveness and Predictability of the System}

Metabolic control analysis [18] deals mainly with the steady state of a system of enzymes that connect series of metabolites. In our view, this theory is not directly applicable in the case of the E1 ubiquitin system composed only of one enzyme, with different enzyme forms. Nevertheless some of the concepts developed for metabolic control analysis could be of application to the E1 system. Hence, the response of the system to changes in the concentration of ATP or ubiquitin was tested. Considering the rate of ubiquitination of Blot (Figure 1, reaction 17) as the final flux, representation of $\ln$ ATP (Figure 7 (a)) or ln Ubiquitin (Figure 7 (b)) versus $\ln$ rate of synthesis of BlotUb, a good correlation among those parameters were obtained showing the responsiveness of the theoretical approach. The apparent elasticity coefficient of E1 towards ATP and ubiquitin were near 0 and 0.29 , respectively at the concentration points used routinely in the assays, $20 \mu \mathrm{M}$ and $6 \mu \mathrm{M}$, respectively.

The predictability of the system was tested with a situation not contemplated in the previous kinetics treatment: the influence of the concentration of AMP on the synthesis of $\mathrm{p}_{4} \mathrm{~A}$ in the presence of ATP and ubiquitin. That influence on AMP, predicted using the Mathematica Program (Figure 7 (c)) was later approached experiment-

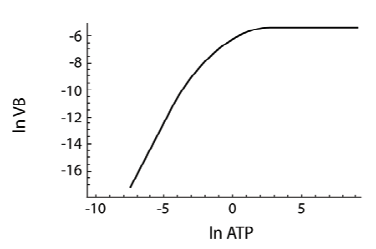

(a)

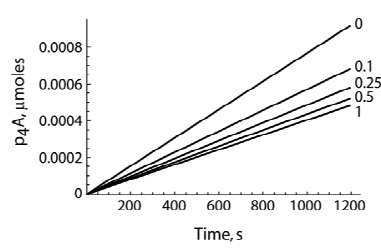

(c)

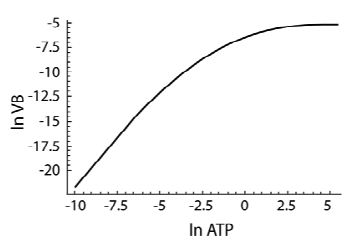

(b)

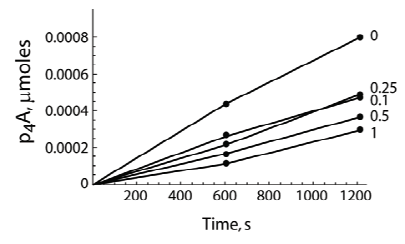

(d)
Figure 7. Responsiveness and predictability of the E1 system. As a measure of the responsiveness of the system, the influence of the concentration of ATP (panel a) or ubiquitin (panel b) on the ubiquitination of Blot are represented as a double-logarithmic plot. The elasticities of E1 towards ATP or ubiquitin are the slopes of the tangents of the corresponding curves. The predictability of the system was tested in two complementary ways. That influence on AMP on the synthesis of $\mathrm{p}_{4} \mathrm{~A}$, predicted using the Mathematica Program (Figure 7 (c)), was later approached experimentally with identical results (Figure 7 (d)). 
tally with identical results (Figure 7 (d)). In both cases AMP accelerated the rate of synthesis of $\mathrm{p}_{4} \mathrm{~A}$. The physiological implications of this finding will be explored elsewhere in another study.

\section{CONCLUDING REMARKS}

The above results may be considered from different perspectives. The ubiquitin activating enzyme $\mathrm{E} 1$ has been treated as a complex enzyme system comprising 8 reactants, 2 inhibitors/effectors, and 19 enzyme forms (EFs) (Figure 1). Calculation of the rate equations of this type of reactions has been approached with the King and Altman method [19] and/or with the simplifications introduced in [20,21] and in [17]. For a general comment on these procedures, see [22]. Although these methods may appear mathematically simple they present great difficulties due to the potential errors introduced during the process of manual operations. Fortunately, with the introduction of computer methods of calculation, performed here with the Mathematica Program [15], treatment of the concerned equations has been greatly simplified. The only difficulty is how to feed the computer with the appropriate information. The simplifications introduced by Cha [17] have been of great help for the mathematical treatment of the postulated reactions catalyzed by the ubiquitin activating enzyme, a system that seemed to be particularly appropriated for this mathematical treatment. Cha [17] postulated that when an enzyme-catalyzed reaction consists of more than one step, and one or more portions of the whole reaction are significantly faster than the over-all reaction, the partial reactions in such segments have almost reached equilibrium when the over-all reaction reaches a steady state. As shown in Figure 1, clusters of reactions of the E1 system involving binding of substrates were considered in rapid equilibrium, were grouped in boxes $\mathrm{X}, \mathrm{Y}$ and $\mathrm{Z}$, and treated as three single entities; another group of reactions involving modification of the bound substrates were treated as in a steady state situation. Altogether, the panorama is similar to the one presented by Cha to develop his methodology [17]. In our view, other complex enzyme situations can be approached in the same way, and the kinetics treatment of E1, here shown, can be taken as an example. Presently we are applying Cha's approach to the mathematical treatment of the signal transductions involved in glycogen metabolism.

Although $\mathrm{P}_{3}$ and $\mathrm{p}_{4} \mathrm{~A}$ are here exclusively considered as a kinetic tool to unravel the set of reactions catalyzed by E1, they also have physiological significance by themselves. Inorganic polyphosphates are probably present in every cell $[23,24,25]$ and are particularly abundant in yeast extracts where $\mathrm{P}_{3}$ and $\mathrm{P}_{4}$ have been described at $\mathrm{mM}$ concentrations [23]. As previously pointed out [3,26], the potential effects exerted by polyphosphates can be considered under two different as- pects: Either caused by their presence at high concentration and frequently condensed in organelles or caused by their occurrence at low concentration in different cellular compartments. Although their role in the last case is not clear, there is a widespread concern about the possibility of polyphosphates playing more general and universal functions in biology $[23,24]$. In the case of E1, it is difficult for us to envisage the physiological role of $\mathrm{P}_{3}$, considering its high apparent $K_{\mathrm{m}}$ value in the reactions here described [3]. The occurrence of $\mathrm{p}_{4} \mathrm{~A}$ has been described in chromaffin granules of the adrenal medulla [27], in skeletal and cardiac muscle [28,29], in the aqueous humor of rabbits [30] and in yeast [31]. $\mathrm{p}_{4} \mathrm{~A}$ has been involved in yeast sporulation [31] and as a modulator of cardiac vascular tone [29]. $\mathrm{p}_{4} \mathrm{~A}$ could also indirectly modulate the levels of $\mathrm{Ap}_{4} \mathrm{~A}$ and $\mathrm{Ap}_{4} \mathrm{~N}$ (nucleotides with apparently elusive physiological roles [32, $33])$, by inhibiting $\left(K_{\mathrm{i}}, \mathrm{nM}\right)$ the activity of the (assymetrical) dinucleoside tetraphoshatase (EC 3.6.1.17) [34].

The present study can also be viewed as pertinent to the so-called System Biology aiming to understand general principles of the complexity of living cells $[35,36$, 37]. This complexity is so astonishing that restricted approaches to simple networks could pave the way for the uncovering of some general principles governing higher organisms. Examples are the study of signal transductions, gene expression, evolution and selforganization, understanding the cell cycle, fluxes in metabolic pathways, neural networks, modeling of biological processes, etc. Study of metabolic processes is one of the main topics of modern System Biology and the work presented here can be considered as an example on how the conjugation of a theoretical and an experimental approach to a particular topic, may help to jump from a mere reductionism to a more global vision of that problem.

The present study has to be considered as an example rather than a framework for the mathematical treatment of complex biological systems. This work may be important to understand the ubiquitin proteasome system itself. This system is the center of a vast arrangement of cellular processes including: cell cycle, transcription control, regulation of enzyme levels, antigen presentation, rate for disposing of unfolded or damaged proteins and controlling life span of regulatory proteins, etc. Alteration of the ubiquitin proteasome system is also responsible for the genesis of malignant diseases and neurodegenerative disorders.

In summary, as shown above this work deals only with the ubiquitin activating enzyme (E1). The reactions catalyzed by E1 are well known from studies developed by Haas, Ciechanover, Hersko, Rose and others $[4,5,6]$, mainly in crude extracts. The present report, greatly inspired by their work, has been carried out with purified, and commercially available E1. The use of $\mathrm{P}_{3}$ as substrate of the enzyme has greatly helped to follow the 
steps of the intermediary reactions (see above). Two mathematical methods have been used for that purpose: Comprehensive and Simplified methods. Some of the kinetic constants involved in these reactions, implicit in the Comprehensive Method, are not yet known and have been tuned; in addition, a simplification of the Comprehensive method has been introduced. The corner-stone of the mathematical treatment of any of these systems is that the experimental and the theoretical results must be consistent. When this is so, the theoretical approach can be used with more confidence and new questions can be theoretically proposed and contrasted with experimental results. In this regard, the effect of potential drugs acting on E1 [38] can be explored in the presence of ATP, $\mathrm{P}_{3}$ and ubiquitin, and the experimental results contrasted with the theoretical approach developed here aiming to pinpoint their precise mode of drug action.

\section{CONCLUSSIONS}

The aim of this work was to investigate the reactions catalyzed by the ubiquitin activating enzyme (E1) using ubiquitin, ATP, adenosine tetraphosphate $\left(\mathrm{p}_{4} \mathrm{~A}\right)$, pyrophosphate, tripolyphosphate, and ubiquitin activating enzyme (E2) as substrates and iodoacetamide dithiothreitol, as inhibitors of the reaction. A total of 19 different hypothetical enzyme forms (EF) of E1 and 22 different enzyme reactions were established and the equations relating the enzyme forms approached with a system of ordinary differential equations and solved with the help of the Mathematica Program. This study can be view a) as a way to treat a complex biological system, b) as a possibility to follow the steps of the intermediate reactions and the theoretical concentrations of the intermediate enzyme forms; c) as a method to study the effects of possible inhibitors on E1 and by extrapolation on the proteasome system itself. Note: the Supplemental Data is available on request from Antonio Sillero at: antonio.sillero@uam.es.

\section{ACKNOWLEDGEMENTS}

This investigation was supported by grants from Dirección General de Investigación Científica y Técnica (BFU 2008-00666 and BFU200908977). We thank Anabel de Diego, Antonio Fernández and Javier Pérez for very capable technical assistance and Professor José Gonzalez Castaño and Dr. Gabriela Morreale de Escobar for helpful suggestions and critical reading of the manuscript.

\section{REFERENCES}

[1] Sillero, A. and Günther Sillero, M.A. (2000) Synthesis of dinucleoside polyphosphates catalyzed by firefly luciferase and several ligases. Pharmacology and Therapeutics, 87, 91-102.

[2] Ortiz, B., Sillero, A. and Günther Sillero, M.A. (1993) Specific synthesis of adenosine(5')tetraphospho(5') nucleoside and adenosine(5')oligophospho(5')adenosine $(\mathrm{n}>4)$ catalyzed by firefly luciferase. European Journal of Biochemistry, 212, 263-270.

[3] Günther Sillero, M.A., de Diego, A., Silles, E. and Sillero, A. (2005) Synthesis of (di)nucleoside polyphosphates by the ubiquitin activating enzyme E1. FEBS Letter, 579, 6223-6229.

[4] Haas, A.L. and Rose, I.A. (1982) The mechanism of ubiquitin activating enzyme. A kinetic and equilibrium analysis. Journal of Biological Chemistry, 257, 1032910337.

[5] Haas, A.L., Warms, J.V., Hershko, A. and Rose, I.A. (1982) Ubiquitin-activating enzyme. Mechanism and role in protein-ubiquitin conjugation. Journal of Biological Chemistry, 257, 2543-2548.

[6] Haas, A.L., Warms, J.V. and Rose, I.A. (1983) Ubiquitin adenylate: Structure and role in ubiquitin activation. Biochemistry, 22, 4388-4394.

[7] Hershko, A. and Ciechanover, A. (1998) The ubiquitin system. Annual review of biochemistry, 67, 425-479.

[8] Baumeister, W., Walz, J., Zuhl, F. and Seemuller, E. (1998) The proteasome: Paradigm of a self-compartmentalizing protease. Cell, 92, 367-380.

[9] Ciechanover, A., Orian, A. and Schwartz, A.L. (2000) The ubiquitin-mediated proteolytic pathway: Mode of action and clinical implications. Journal of Cell Biochemistry Supply, 34, 40-51.

[10] Glickman, M.H. and Ciechanover, A. (2002) The ubiquitin-proteasome proteolytic pathway: Destruction for the sake of construction. Physiological reviews, 82, 373-428.

[11] Lee, I. and Schindelin, H. (2008) Structural insights into E1-catalyzed ubiquitin activation and transfer to conjugating enzymes. Cell, 134, 268-278.

[12] Selivanov, V.A., Puigjaner, J., Sillero, A., Centelles, J.J., Ramos-Montoya, A., Lee, P.W. and Cascante, M. (2004) An optimized algorithm for flux estimation from isotopomer distribution in glucose metabolites. Bioinformatics, 20, 3387-3397.

[13] Torrecilla, A., Marques, A.F., Buscalioni, R.D., Oliveira, J.M., Teixeira, N.A., Atencia, E.A., Günther Sillero, M.A. and Sillero, A. (2001) Metabolic fate of AMP, IMP, GMP and XMP in the cytosol of rat brain: An experimental and theoretical analysis. Journal of Neurochemistry, 76, 1291-1307.

[14] Osorio, H., Carvalho, E., del Valle, M., Günther Sillero, M.A., Moradas-Ferreira, P. and Sillero, A. (2003) $\mathrm{H}_{2} \mathrm{O}_{2}$, but not menadione, provokes a decrease in the ATP and an increase in the inosine levels in Saccharomyces cerevisiae. An experimental and theoretical approach. European Journal of Biochemistry, 270, 1578-1589.

[15] Wolfram, S. (1996) The Mathematica Book. Cambridge University Press, Cambridge.

[16] Segel, I.H. (1975) Enzyme kinetics: Behavior and analysis of rapid equilibrium and steady state enzyme systems. Wiley, New York.

[17] Cha, S. (1968) A simple method for derivation of rate equations for enzyme-catalyzed reactions under the rapid equilibrium assumption or combined assumptions of equilibrium and steady state. Journal of Biological Chemistry, 243, 820-825.

[18] Fell, D. (1997) Understanding the control of metabolism, Portland Press, MiamiBrookfield. 
[19] King, E.L. and Altman, C. (1956) A schematic method for deriving the rate laws for enzyme-catalyzed reactions. The Journal of Physical Chemistry, 60, 1357-1378.

[20] Wong, J.T. and Hanes, C.S. (1962) Kinetic formulations for enzymic reactions involving two substrates. Canadian journal of biochemistry and Physiology, 40, 763-804.

[21] Volkenstein, M.V. and Goldstein, B.N. (1966) Allosteric enzyme models and their analysis by the theory of graphs. Biochimica et Biophysica Acta, 115, 471-477.

[22] Cornish-Bowden, A. (1995) Fundamental of enzyme kinetics. Portland Press, London.

[23] Kulaev, I.S. and Vagabov, V.M. (1983) Polyphosphate metabolism in micro-organisms. Advances in Microbial Physiology, 24, 83-171.

[24] Wood, H.G. and Clark. J.E. (1988) Biological aspects of inorganic polyphosphates. Annual Review of Biochemistry, 57, 235-260.

[25] Kornberg, A., Rao, N.N. and Ault-Riche, D. (1999) Inorganic polyphosphate: A molecule of many functions. Annual Review of Biochemistry, 68, 89-125.

[26] Günther Sillero, M.A., de Diego, A., Silles, E., Osorio, H. and Sillero, A. (2003) Polyphosphates strongly inhibit the tRNA dependent synthesis of poly(A) catalyzed by poly(A) polymerase from Saccharomyces cerevisiae. FEBS Letter, 550, $41-45$.

[27] Günther Sillero, M.A., Del Valle, M., Zaera, E., Michelena, P., Garcia, A.G. and Sillero, A. (1994) Diadenosine 5',5'-P1,P4-tetraphosphate $\left(\mathrm{Ap}_{4} \mathrm{~A}\right)$, ATP and catecholamine content in bovine adrenal medulla, chromaffin granules and chromaffin cells. Biochimie, 76, 404-409.

[28] Small, G.D. and Cooper, C. (1966) Studies on the occurrence and biosynthesis of adenosine tetraphosphate. Biochemistry, 5, 26-33.

[29] Westhoff, T., et al. (2003) Identification and characterization of adenosine 5'-tetraphosphate in human myocar- dial tissue. The Journal of Biological Chemistry, 278, 17735-17740.

[30] Pintor, J., Pelaez, T. and Peral, A. (2004) Adenosine tetraphosphate, Ap4, a physiological regulator of intraocular pressure in normotensive rabbit eyes. Journal of Pharmacology and Experimental Therapeutics, 308, 468-473.

[31] Jakubowski, H. (1986) Sporulation of the yeast Saccharomyces cerevisiae is accompanied by synthesis of adenosine 5'-tetraphosphate and adenosine 5'-pentaphosphate. Proceedings of the National Academy of Sciences, 83, 2378-2382.

[32] McLennan, A.G. (2000) Dinucleoside polyphosphatesfriend or foe? Pharmacology and Therapeutics, 87, 73-89.

[33] Mc Lennan, A.G., et al. (2001) Recent progress in the study of the intracellular functions of diadenosine polyphosphates. Drug Development Research, 52, 249-259.

[34] Lobatón, C.D., Vallejo, C.G., Sillero, A. and Sillero, M.A. (1975) Diguanosinetetraphosphatase from rat liver: Acitivity on diadenosine tetraphosphate and inhibition by adenosine tetraphosphate. European journal of Biochemistry, 50, 495-501.

[35] Alon, U. (2006) An introduction to system biology. Design principles of biological circuits. CRC Press, Taylor \& Francis Group, London.

[36] Klipp, E., Herwig, R., Kowald, A., Wierling, C. and Lehrach, H. (2005) System biology in practice. Concepts, implementation and application, Wiley-VCH Verlag GmbH \& Co., Weinhein.

[37] Fell, D. (1997) Undersatnding the control of metabolism, Portland Press, London.

[38] Yang, Y., et al. (2007) Inhibitors of ubiquitin-activating enzyme (E1), a new class of potential cancer therapeutics. Cancer Research, 67, 9472-9481. 